\title{
La percepción cognitiva de los recursos naturales y medioambientales como factor interviniente en la imagen afectiva del destino turístico
}

\author{
Lucio Hernández-Lobato* \\ María Magdalena Solís-Radilla \\ Héctor Tomás Pastor-Durán \\ Unidad Académica de Turismo \\ Universidad Autónoma de Guerrero
}

\section{Resumen}

El objetivo de este trabajo es analizar la imagen percibida de un destino turístico de playa, en particular los recursos naturales y medioambientales de la dimensión cognitiva y su papel en la conformación de la dimensión afectiva de la imagen, así como las fortalezas y debilidades del destino. Se utilizó la técnica estructurada para analizar el componente común de la imagen del destino turístico por medio de la percepción de los turistas acerca de cada uno de los atributos, a través de escalas tipo Likert y diferencial semántico. A partir del márketing turístico, y desde la perspectiva de la demanda, se plantea una influencia directa y positiva de los recursos naturales y medioambientales de la dimensión cognitiva sobre la dimensión afectiva de la imagen del sitio, y se prueba en un estudio empírico con turistas nacionales en Acapulco, Guerrero, México. Los resultados confirman además que, en general, los atributos de la imagen cognitiva se encuentran posicionados favorablemente, siendo los más débiles los referentes a la limpieza e higiene y al grado de urbanización.

\section{Palabras clave}

Turismo, márketing, imagen del destino turístico, medioambiente, percepción.

Recibido: 10/12/2014 · Aceptado: 10/03/2015

*Correo electrónico: luciohernandez2010@gmail.com 


\title{
Cognitive perception of natural and environmental resources as an intervening factor in affective image of the touristic destination
}

\begin{abstract}
The aim of this work is to analyze the perceived image of a beach resort, particularly the factors of natural and environmental resources in a cognitive dimension and its participation in shaping the affective dimension of the image as well as the its strengths and weaknesses. A structured technique was used to analyze the common component of the image of the destination through the perception that tourists have of each of the attributes, applying Likert scales and semantic differential. Within tourism marketing and from the demand perspective, a direct and positive influence of the cognitive dimension of natural and environmental resources upon the affective dimension of the destination image is proposed; this is proven through an empirical study of national tourists in Acapulco, Guerrero, Mexico. In addition, results confirm that in general, the attributes of the cognitive dimension are favorably established with the weaker aspects related to the city's cleanliness and hygiene and the degree of urbanization.
\end{abstract}

\section{KEY WORDS}

Tourism, marketing, destination image, environment, perception. 


\section{Introducción}

En la investigación científica el estudio de la actividad turística conlleva una serie de peculiaridades susceptibles de ser analizadas, una de ellas es la imagen percibida por los turistas que visitan un destino de sol y playa, tema que durante las últimas décadas ha despertado un creciente interés en los estudiosos del márketing y su ámbito de aplicación en el turismo. De acuerdo con un análisis bibliométrico de Bigné et al. (2008), los subsectores más examinados son los destinos turísticos y los tipos de turismo; sin embargo, las investigaciones dedicadas al segmento de sol y playa son minoritarios, de ahí el interés de ahondar al respecto.

Sobre la imagen de los destinos se han estudiado diversos factores (accesibilidad, cultura, seguridad, vida nocturna y entretenimiento, relación calidad/ precio y el factor afectivo), el que nos interesa aquí es el de los recursos naturales y el medioambiente, el cual, a partir de la preocupación por el vínculo entre turismo y sustentabilidad, obliga a asumir nuevos comportamientos ante las necesidades que emergen referentes a los temas conservacionistas, ya que el concepto de destino turístico está fuertemente ligado a las condiciones geográficas y medioambientales de un lugar.

El atractivo de una plaza turística depende de manera significativa de los recursos naturales y las condiciones climáticas y ambientales percibidos en la imagen proyectada (Govers, 2005), de modo que se convierten en un elemento básico en la toma de decisión final, en la repetición de la visita y en la recomendación a otros (Solís et al., 2014) por parte del turista de primera vez o de aquellos que regresan y cuentan con una experiencia en el destino, ya que la información obtenida de primera mano puede modificar o cambiar la imagen ya establecida (Echtner y Ritchie, 2003).

Ya no solo habrá que enfrentar retos como la internacionalización de las empresas turísticas, la estacionalidad del sector, la desconcentración geográfica, los nuevos productos alternativos y las incorporaciones tecnológicas, sino también la necesaria consolidación de un turismo de calidad apoyado en un medioambiente sostenible, que influya en la generación de sentimientos positivos hacia el sitio y que se transforma en el principal reto de quienes participan en el desarrollo y la gestión de este sector (Prados, 2002). 


\section{Objetivos de la investigación}

Objetivo general de la investigación

Analizar la participación de los recursos naturales y medioambientales en la conformación de la dimensión cognitiva de la imagen del destino turístico como un factor interviniente en la dimensión afectiva, desde la perspectiva del turista nacional que realiza su experiencia turística en Acapulco, Guerrero, México.

Objetivos específicos

Identificar las fortalezas y debilidades de los atributos de la imagen del destino turístico.

Caracterizar el perfil de los turistas nacionales que visitan el destino.

\section{Imagen del destino turístico}

Desde el momento en que se promueve el desarrollo turístico en un lugar hasta la fase de puesta en marcha, los recursos naturales (principal fuente de atracción turística) son los que reciben los impactos de la propia actividad, con los consecuentes desequilibrios ecológicos que esto genera. Al tratarse de un recurso limitado se hace imprescindible reconocer su preservación a través de un seguimiento permanente de los impactos que sufre, para tomar decisiones que mejoren el desarrollo de la actividad turística, ya que, de acuerdo con Vera y Baños (2010), los lugares turísticos de litoral muestran inconsistencias respecto de la competitividad y de su capacidad de adaptación a las tendencias del mercado turístico, y son los que en mayor medida manifiestan los efectos negativos del desarrollo de hoteles, marinas, centros de diversión, campos de golf, etc., cuyo uso del suelo, agua, aire y de la biodiversidad atenta contra el medio ambiente (San Martín Rebolloso y Salcedo, 2007).

Después de analizar los hallazgos en diversos estudios en psicología ambiental, Baloglu y Brinberg (1997) sostienen que los ambientes y los lugares tienen imágenes tanto cognitivas como afectivas, y que la imagen global de un sitio se compone tanto de elementos designativos como apreciativos, los cuales son tratados en estudios de márketing y comportamiento del consumidor bajo las etiquetas creencias y afecto, como se observa en algunos de los trabajos de 
márketing turístico más citados (Baloglu y Brinberg, 1997; Baloglu y McCleary, 1999; Vogt y Andereck, 2003), que identifican dos componentes ligados de los que se deriva la interpretación o entendimiento de la imagen: la razón y los sentimientos. Es decir, las evaluaciones perceptuales/cognitivas tienen que ver con las creencias y los conocimientos de los turistas acerca de los diferentes atributos del destino, y las afectivas se vinculan con los sentimientos que el destino inspira en el turista.

Entonces, existe consenso en que la percepción de la imagen del destino turístico se manifiesta en tres dimensiones: cognitiva (perceptual), afectiva (sentimientos) y global, aunque no siempre sean utilizadas simultáneamente para medir la imagen (Beerli, Martín y Moreno, 2003; Vogt y Andereck, 2003; Beerli y Martín, 2004a, 2004b).

En la literatura se observa una gran cantidad de definiciones o acercamientos al concepto de imagen del destino turístico. Según Gartner (1993), estas imágenes se desarrollan con tres componentes interrelacionados de manera jerárquica: cognitivos, afectivos e intencionales. Para Luque et al. (2004), la imagen de un destino puede definirse como una red de elementos: una estructura de conocimiento que sintetiza lo que conocemos acerca de cierto lugar y los sentimientos que este provoca.

En el presente trabajo se aborda el concepto de imagen centrado en las percepciones del cliente-consumidor, es decir, se define como la valoración que hace el turista de los atributos funcionales-psicológicos del destino (imagen cognitiva) y sus sentimientos o respuestas afectivas manifestadas hacia los lugares (el componente afectivo de la imagen) (Beerli y Martín, 2004a), los cuales son desarrollados a través de experiencias vinculadas con el lugar, sus habitantes y los objetos y organizaciones asociadas con el mismo (Del Barrio García, Luque Martínez y Rodríguez Molina, 2009). Destaca la participación del factor recursos naturales y medioambientales en la configuración de la imagen cognitiva (IC), entendida como un constructo evaluativo y multidimensional que interviene en la imagen afectiva (IA) hacia el destino, por eso la evaluación global puede variar de una persona a otra y de una región o país a otro, dependiendo de las fuentes de información (estímulos) a las que se tenga acceso y a la influencia de aspectos (personales) motivacionales y demográficos. 
Acapulco, destino de litoral maduro

Acapulco se encuentra dentro de los llamados destinos tradicionales de sol y playa en el Pacífico mexicano; tuvo su despegue en el contexto internacional durante la década de los sesenta y en los últimos años se ha convertido prácticamente en la principal plaza de litoral del turismo nacional y de segunda residencia. De acuerdo con Camisón y Monfort (1998), puede considerarse un destino en etapa de madurez y con posibles rasgos negativos en la gestión medioambiental, por lo que está obligado a poner en marcha políticas de renovación y reestructuración basadas en los elementos del sistema turístico (López, 2014). Por lo anterior se plantea la siguiente hipótesis del trabajo:

$\mathrm{H}_{1}$ Existe una influencia directa y positiva de los recursos naturales y medioambientales como un factor de la dimensión cognitiva sobre la dimensión afectiva de la imagen de Acapulco en cuanto destino turístico de sol y playa.

\section{Metodología}

Diseño de la escala

La especificación del dominio y la generación de ítems se basaron en la revisión de la literatura sobre la imagen de destinos turísticos, la cual también permitió identificar como punto de partida un conjunto amplio de ítems agrupados en torno a varios factores básicos de la imagen. Se realizó una consulta entre académicos especializados en mercadotecnia y turismo de las universidades de Valencia, Jaume I de Castellón, en España, y de la Universidad Autónoma de Guerrero, en México, para eliminar los ítems con menor grado de acuerdo; después se llevó a cabo una preprueba con 20 turistas en el propio puerto de Acapulco, sin la necesidad de modificar ninguno, salvo alguna corrección en la redacción. Con esta primera reducción, la escala quedó formada por seis factores de la imagen cognitiva y uno de la afectiva. Sin embargo, para efecto del presente análisis solo se consideró el factor relativo a los recursos naturales y medioambientales de la dimensión cognitiva y la dimensión afectiva (cuadro 1).

La información se obtuvo mediante un cuestionario que se aplicó personalmente a cada turista nacional durante su estancia vacacional en el puerto. Se utilizó la técnica estructurada ya que, en concordancia con los objetivos planteados, se pretendía analizar el componente común de la imagen del 


\section{Cuadro 1. Conjunto de ítems de la escala imagen del destino turístico}

\section{DimENSIÓN COGNITIVA}

1. Tiene un clima agradable

\section{Estudios CONSIDERADOS PARA LA SELECCIÓN DE ÍTEMS*}

Hu y Ritchie (1993); Court y Lupton (1997); Murphy (1999); Chen y Hsu (2000); Baloglu y Mangaloglu (2001); Bigné y Sánchez (2001); Beerli, Martín y Moreno (2003); Hui y Wan (2003); O’Leary y Deegan (2003); Pike (2003, 2007); Domínguez, Valdés y Morfín (2004); Hsu, Wolfe y Kang (2004); Konecnik (2004, 2005); Pike y Ryan (2004); Wong (2004); Bonn, Joseph y Dai (2005); Castaño, Moreno y Crego (2006); San Martín, Rodríguez del Bosque y Vázquez (2006); Konecnik y Gartner (2007); Sánchez-García et al. (2007); Nadeau et al. (2008).

2. Ofrece mucho en términos de belleza natural escénica

Kale y Weir (1986); Phelps (1986); Chon (1991); Hu y Ritchie (1993); Goodrich (1977, 1978); Kim (1998); Andreu, Bigné y Cooper (2000); Chen y Hsu (2000); Tapachai y Waryszak (2000); Baloglu y Mangaloglu (2001); Bigné y Sánchez (2001); Rittichainuwat, Qu y Brown (2001); O’Leary y Deegan (2003); Pike (2003, 2007); Domínguez, Valdés y Morfín (2004); Hsu, Wolfe y Kang (2004); Konecnik (2004, 2005); Wong (2004): Grosspietsch (2006); San Martín, Rodríguez del Bosque y Vázquez (2006); Hosany, Ekinci y Uysal (2007); Konecnik y Gartner (2007); Tasci, Gartner y Cavusgil (2007); Nadeau et al. (2008).
3. Posee playas de gran calidad y belleza
Phelps (1986); Murphy (1999); Baloglu y Mangaloglu (2001); Bigné y Sánchez (2001); Beerli, Martín y Moreno (2003); Pike (2003, 2007); Konecnik (2004, 2005); San Martín, Rodríguez del Bosque y Vázquez (2006); Konecnik y Gartner (2007).

Baloglu y Mangaloglu (2001); Bigné y Sánchez (2001); O’Leary y Deegan (2003); Konecnik (2004, 2005). conservación

medioambiental

5. Tiene un buen grado de urbanización

6. Posee un elevado nivel de limpieza e higiene

Dimensión AFECTiva

1. Desagradable-agradable

2. Aburrido-estimulante

3. Angustiante-relajante

4. Triste-alegre
Bigné y Sánchez (2001); O’Leary y Deegan (2003); Konecnik (2004); Sanchez-García et al. (2007).

Kim (1998); Bigné y Sánchez (2001); Joppe, Martin y Waalen (2001); Hui y Wan (2003); O’Leary y Deegan (2003); Konecnik (2004, 2005); Wong (2004); Bonn, Joseph y Dai (2005); Castaño, Moreno y Crego (2006); Sánchez-García et al. (2007); Hosany, Ekinci y Uysal (2007); Lee et al. (2010).

Estudios CONSIDERAdos PARA LA SELECCIÓN DE ÍTEMS*

Baloglu y Brinberg (1997); Baloglu y McCleary (1999); Baloglu (2001); Baloglu y Mangaloglu (2001); White (2002); Kim y Richardson (2003); Beerli y Martín (2004a, 2004b); Pike y Ryan (2004); Konecnik (2005); Ekinci y Hosany (2006); Hong et al. (2006); San Martín, Rodríguez del Bosque y Vázquez (2006); Hosany, Ekinci y Uysal (2006, 2007); Lee et al. (2010).

* Ítems retomados y adaptados de estos estudios, entre otros.

Fuente: Elaboración propia. 
destino turístico, que consiste en el agrupamiento de atributos de la imagen, y por medio del cual se recoge la percepción de los turistas.

En cuanto a los atributos cognitivos, a través de una escala Likert de cinco posiciones, los turistas indicaron su grado de acuerdo o desacuerdo con los seis ítems planteados ( 1 = totalmente en desacuerdo; 2 = en desacuerdo; 3 = ni de acuerdo ni en desacuerdo; 4 = de acuerdo; 5 = totalmente de acuerdo). A su vez, las cuatro escalas definidas para la pregunta ¿qué sentimiento experimenta usted al pensar en este destino turístico? fueron: desagradable-agradable, aburrido-estimulante, angustiante-relajante y triste-alegre, que miden los atributos afectivos a través de escalas bipolares de diferencial semántico, con cinco posiciones ( 1 = muy; 2 = bastante; 3 = ni uno ni otro; 4 = bastante; 5 = muy), ya que según Dolnicar y Grün (2013) los formatos con opciones de respuesta totalmente verbalizados muestran un mejor funcionamiento.

Se trabajó con un método de muestreo no probabilístico. Con base en la propuesta de Kozak (2001), la muestra se conformó por aquellos individuos mayores de 18 años que llevaban el tiempo suficiente para aportar datos fiables sobre la percepción del destino turístico. Para evitar la repetición o imitación en las respuestas y obtener diferentes puntos de vista en la recolección de datos, se aplicó el cuestionario en diversos puntos de atracción o de afluencia turística (playas, plazas, terminales de transporte, hoteles, restaurantes), y solo a una persona por cada familia o grupo de turistas nacionales que por motivos de ocio o vacaciones llevaban al menos tres días en Acapulco. Tras obtener y depurar la información, se obtuvo un total de 440 cuestionarios válidos.

\section{Resultados y discusión}

Análisis descriptivo

Se realizó un análisis descriptivo que incluye dos apartados: el perfil de los turistas nacionales que visitan Acapulco como destino de playa y los elementos inherentes a la variable imagen en donde se analizan los valores medios de los ítems considerados en el estudio. 


\section{Perfil del turista nacional}

El rango de las edades significativamente superiores es de 25-34 hasta 45-54 años, con casi $60 \%$ de la muestra, lo que revela claramente que se trata de un segmento en la demanda de adultos-jóvenes, pues la edad promedio es de casi 37 años. Los estados más cercanos y colindantes (Estado de México, Morelos, Jalisco, Michoacán, Guanajuato, Puebla) son los que indican su preferencia para visitar Acapulco, no obstante, el Distrito Federal es el principal mercado actual, con $45.5 \%$ de la demanda nacional. En cuanto a las visitas previas a Acapulco, se observa un comportamiento repetitivo en $25.9 \%$ de los turistas que lo han visitado diez o más veces; la visita previa promedio es de casi cuatro ocasiones (3.94). Lo anterior confirma los resultados de la mayoría de los estudios realizados en este sentido, ya que Acapulco es considerado el primero y más destacado entre los destinos tradicionales de México. Por lo que toca a los días de estancia, los resultados descubren que de tres a cinco días son los que abarcan el mayor porcentaje (69.4\%), y al analizar los valores medios se corrobora lo descrito: la estancia promedio es un poco mayor a cinco días (5.25) (cuadro 2).

Cuadro 2. Descripción de la muestra por días de estancia y visitas previas

\begin{tabular}{|c|c|c|c|c|c|}
\hline \multicolumn{6}{|c|}{ ACAPULCO } \\
\hline DÍAS DE ESTANCIA & $\mathrm{Fr}$ & $\%$ & VISITAS PREVIAS & $\mathrm{Fr}$ & $\%$ \\
\hline 3 & 102 & 23.2 & Ninguna & 42 & 9.5 \\
\hline 4 & 91 & 20.7 & 1 & 45 & 10.2 \\
\hline 5 & 113 & 25.7 & 2 & 74 & 16.8 \\
\hline 6 & 29 & 6.6 & 3 & 52 & 11.8 \\
\hline 7 & 47 & 10.7 & 4 & 30 & 6.8 \\
\hline 8 & 30 & 6.8 & 5 & 31 & 7.2 \\
\hline 9 & 3 & 0.7 & 6 a 9 & 52 & 11.8 \\
\hline 10 o más & 25 & 5.6 & 10 o más & 114 & 25.9 \\
\hline Total & 440 & 100.0 & Total & 440 & 100.0 \\
\hline & \multicolumn{2}{|c|}{ Media } & & \multicolumn{2}{|c|}{ Media } \\
\hline Días de estancia & \multicolumn{2}{|c|}{5.25} & Visitas previas & \multicolumn{2}{|c|}{3.94} \\
\hline
\end{tabular}

Fuente: Elaboración propia. 


\section{Valores medios de los atributos de la imagen}

Las valoraciones medias (cuadro 3) que se refieren a la importancia de cada atributo del factor recursos naturales y medioambientales, correspondientes a la imagen cognitiva, se encuentran por encima del punto neutral; las principales fortalezas de este destino turístico de playa son las siguientes: "Tiene un clima agradable”, "Ofrece mucho en términos de belleza natural escénica”, "Posee playas de gran calidad y belleza” y "Existe una elevada conservación medioambiental”.

Cuadro 3. Valores medios de la imagen cognitiva

\begin{tabular}{llc}
\multicolumn{1}{c}{ Clave } & \multicolumn{1}{c}{ ÍtEms } & Medias \\
Clima* & Tiene un clima agradable & 4.48 \\
Natural & Ofrece mucho en términos de belleza natural escénica & 4.25 \\
Playas & Posee playas de gran calidad y belleza & 4.07 \\
Medioambiental & Existe una elevada conservación medioambiental & 4.38 \\
Urbaniza & Tiene un buen grado de urbanización & 3.79 \\
Limpieza & Posee un elevado nivel de limpieza e higiene & 3.20 \\
\hline * Este ítem será eliminado de la escala tras realizar los análisis de dimensionalidad, fiabilidad y \\
validez
\end{tabular}

A pesar de que no se encontraron niveles con tendencia negativa (menores de tres) en los atributos, hay que señalar que el concerniente a la limpieza e higiene del lugar se manifiesta como el más débil, seguido por el grado de urbanización, por ello habrá que ponerles especial atención para mejorar la imagen cognitiva.

Como puede observarse en el cuadro 4, los vínculos afectivos (IA) que el destino de playa despierta en sus visitantes también se encuentran por arriba del punto neutral y son lo suficientemente altos para decir que Acapulco es percibido como un lugar entre bastante y muy alegre (4.85), agradable y estimulante (4.73), sin dejar de ser relajante (4.64), aunque en menor medida. 
Cuadro 4. Valores medios de la imagen afectiva

\begin{tabular}{llr}
\multicolumn{1}{r}{ Clave } & \multicolumn{1}{c}{ Ítems } & Medias \\
\hline Desagrado & Desagradable-agradable & 4.73 \\
Aburrido & Aburrido-estimulante & 4.73 \\
Angustia & Angustiante-relajante & 4.64 \\
Triste & Triste-alegre & 4.85
\end{tabular}

Fuente: Elaboración propia.

\section{Dimensionalidad, fiabilidad y validez de las escalas}

Antes de contrastar la hipótesis se procedió al análisis de dimensionalidad, fiabilidad y validez. Las escalas utilizadas cumplen la validez de contenido, en la medida en que se tuvieron en cuenta todos los aspectos considerados en la literatura para medir la imagen afectiva y cognitiva de un destino turístico, además de que se depuraron los ítems durante el proceso de consulta, lo que dio como resultado lo mostrado en el cuadro 1.-

De acuerdo con Medina, Rey y Rufín (2010), se realizó un análisis factorial exploratorio de componentes principales, el cual determinó que las variables se agrupaban en los factores planteados desde el inicio. Así también, con base en la primera fase del procedimiento propuesto por Anderson y Gerbing (1988), se validaron las escalas de medición mediante un análisis factorial confirmatorio, que ratificó la existencia de una dimensión afectiva y otra cognitiva de la imagen. El modelo obtenido del análisis factorial confirmatorio es adecuado pues la probabilidad de chi cuadrado asociada al mismo es superior a 0.05 (0.1834). La validez convergente se determina porque se constituyen en un solo factor, también se hizo mediante la correlación bivariada entre parejas de ítems. Esta validez se verificó debido al alto valor que toma el alfa de Cronbach de los constructos analizados.

Para el análisis de la fiabilidad se utilizaron tres criterios de depuración definitiva de las escalas, es decir, se eliminaron aquellos ítems que no cumplieron alguno de los criterios siguientes: las cargas factoriales inferiores a 0.6 (Bagozzi y Yi, 1988; Hair et al., 2006), el alpha de Cronbach inferior a 0.80, y la varianza extraída promedio (Average Variance Extracted, AvE) inferior a 0.5 (Fornell y 
Larcker, 1981). En el análisis factorial confirmatorio se comprobó la necesidad de eliminar alguno de los ítems considerados al inicio (cuadro 3), en concreto el ítem: "Tiene un clima agradable" (0.52), incluido dentro de la escala que mide los recursos naturales y medioambientales de la imagen cognitiva.

La validez convergente queda demostrada por dos motivos: porque las cargas factoriales son significativas y superiores a 0.6 (Bagozzi y Yi, 1988; Hair et al., 2006), y porque la Ave para cada factor es siempre superior a 0.5 (Fornell y Larcker, 1981).

La fiabilidad de la escala (cuadro 5) se demuestra porque los índices de fiabilidad compuesta de cada dimensión obtenida son superiores a 0.6 (Bagozzi y Yi, 1988).

Cuadro 5. Análisis de fiabilidad de las escalas de medida (modelo depurado)

\begin{tabular}{|c|c|c|c|}
\hline & Alpha de CRONBACH & $\begin{array}{l}\text { FIABILIDAD COMPUESTA } \\
\text { (IFC) }\end{array}$ & $\begin{array}{l}\text { VARIANZA EXTRAÍDA } \\
\text { PROMEDIO (AVE) }\end{array}$ \\
\hline $\begin{array}{l}\text { Recursos naturales y } \\
\text { medioambientales }\end{array}$ & 0.82 & 0.83 & 0.67 \\
\hline Imagen afectiva & 0.80 & 0.93 & 0.76 \\
\hline
\end{tabular}

Fuente: Elaboración propia.

La validez discriminante de los constructos considerados en el modelo se evalúa a través de la Ave (Fornell y Larcker, 1981). Un constructo debe compartir más varianza con sus indicadores que con otros constructos del modelo, esto sucede cuando la raíz cuadrada del Ave entre cada par de factores es superior a la correlación estimada entre los factores, tal como sucede en el presente caso, por lo que se ratifica su validez discriminante (cuadro 6).

Cuadro 6. Análisis de las escalas (validez discriminante)*

\begin{tabular}{lcc} 
& $\begin{array}{c}\text { Recursos naturales y } \\
\text { medioambientales }\end{array}$ & Imagen afectiva \\
\hline Recursos naturales y medioambientales & 0.82 & 0.82 \\
\hline Imagen afectiva & 0.65 & 0.82 \\
\hline
\end{tabular}

* Debajo de la diagonal: correlación estimada entre los factores.

Diagonal: raíz cuadrada de la varianza extraída.

Fuente: Elaboración propia. 
Teniendo en cuenta las cargas factoriales y los criterios mencionados, el factor recursos naturales y medioambientales de la dimensión cognitiva de la imagen quedó formado por cinco ítems:

RNM1: Existe una elevada conservación medioambiental (0.80).

RNM2: Ofrece mucho en términos de belleza natural escénica (0.70).

RNM3: Posee playas de gran calidad y belleza (0.62).

RNM4: Posee un elevado nivel de limpieza e higiene (0.75).

RNM5: Tiene un buen grado de urbanización (0.72).

A su vez, la dimensión afectiva de la imagen quedó integrada por cuatro ítems:

IA1: Desagradable-agradable (0.88).

IA2: Aburrido-estimulante (0.92).

IA3: Angustiante-relajante (0.83).

IA 4: Triste-alegre (0.91).

\section{Contrastación de la hipótesis}

De acuerdo con el enfoque de dos etapas (two-step) de Anderson y Gerbing (1988), una vez que se ha estimado el modelo de medición estima el modelo estructural aplicando el análisis path con variables latentes, con el que se contrasta la hipótesis planteada, relativa a la influencia de la dimensión cognitiva de la imagen sobre la dimensión afectiva. En la figura 1 se muestra el resultado del modelo estructural; se observa que el ajuste del modelo es adecuado, ya que la probabilidad asociada al estadístico chi cuadrado es superior a $0.05(0.1098)$. Además, el efecto de la dimensión afectiva sobre la cognitiva es de 0.07 , lo que es significativo según los resultados de otros investigadores (San Martín y Rodríguez del Bosque, 2008; Sancho y Álvarez, 2010; Lee et al., 2010), por lo que se confirma la tendencia y se da por aceptada la hipótesis planteada. 


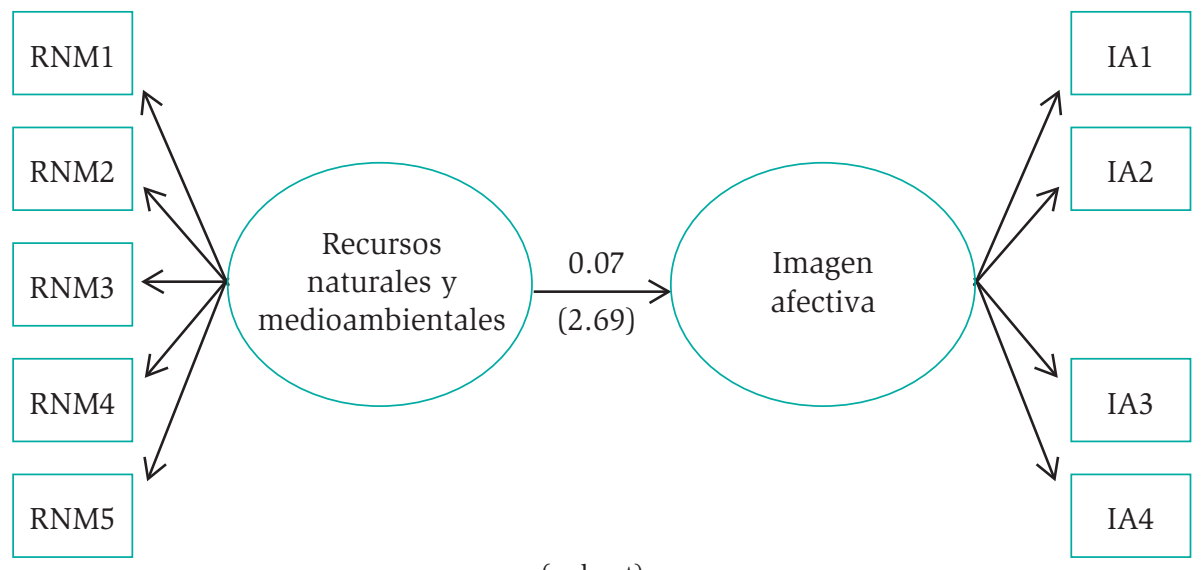

(valor-t)

Ajuste del modelo: $\chi^{2}=35.0863 ; \mathrm{gl}=26 ; \mathrm{P}=0.1098$; rmsea $=0.021$; gfi $=0.995$; agfi $=0.989$. Fuente: Elaboración propia.

Figura 1. Modelos con la relación causal

\section{Conclusiones}

La imagen del destino se ha convertido en uno de los temas que generan mayor interés dentro de la investigación en el campo del turismo y el márketing. Pueden resaltarse tres aspectos clave en su estudio: concepto y naturaleza, formación y su influencia en el comportamiento del turista. De esta manera, concluimos que la imagen del destino turístico es una valoración mental que se forma a partir de la percepción cognitiva y afectiva de los atributos de un conjunto de productos y servicios contenidos por límites geográficos, gestionados y comercializados por organismos públicos y privados, y que por diversos atractivos y motivaciones consiguen generar desplazamientos turísticos. Este constructo evaluativo y multidimensional puede variar de una persona a otra y de una región o país a otro, dependiendo de las fuentes de información a las que se tenga acceso y a la influencia de aspectos motivacionales y demográficos; asimismo, debe concebirse como la percepción del individuo acerca de las características del destino turístico. La revisión bibliográfica evidenció un gran número de estudios que posicionan la imagen del destino turístico como un 
factor determinante al momento de seleccionar el sitio a visitar; en su mayoría, estos estudios han mostrado interés por el aspecto cognitivo de la imagen.

Debido a que el cambio en los grados de atracción y conocimiento del destino durante unas vacaciones puede ser evaluado utilizando percepciones de la imagen, queda de manifiesto el incremento en el conocimiento durante el curso del viaje, por lo que la técnica estructurada brindó un instrumento que demostró que la imagen del destino es un fenómeno multidimensional, de naturaleza cognitiva y afectiva, que permitió medir la imagen que los visitantes perciben de los destinos turísticos y que utilizan para decidir a cuál de ellos viajar.

En cuanto al análisis descriptivo de los resultados obtenidos para la medición de la imagen que proyecta el destino, se observa que la imagen global de Acapulco tiene una clara tendencia favorable o positiva, ya que no se recibieron opiniones negativas o por debajo de los valores medios.

En concordancia con la imagen global de Acapulco, se observa que los niveles relativos a la importancia de cada atributo de la imagen cognitiva se posicionan en general favorablemente: sus principales fortalezas son los recursos naturales y medioambientales, en particular los factores siguientes: tiene un clima agradable, existe una elevada conservación medioambiental, ofrece mucho en términos de belleza natural escénica y posee playas de gran calidad y belleza, en ese orden. Respecto a la imagen afectiva, el puerto se percibe como un lugar de muy a bastante alegre, agradable y estimulante, sin dejar de ser relajante.

Con base en los resultados obtenidos, y a pesar de que no se recibieron opiniones negativas acerca de sus atributos, se sugiere que los organismos encargados de tomar decisiones estratégicas y de márketing en los tres niveles de gobierno pongan especial atención a los atributos con mayor debilidad (la limpieza e higiene del lugar, además de su grado de urbanización, que se manifiesta como el más débil), para mejorar el destino como producto turístico e intentar diferenciarlo con el objetivo de dejar de ser una plaza más en el actual mercado de playa competitivo y formar parte del pequeño grupo de sitios que el turista elige visitar como un destino sustentable.

Respecto a la hipótesis planteada, se da por aceptada debido a que el factor referente a los recursos naturales y medioambientales de la dimensión cognitiva mostró una relación directa y positiva con la dimensión afectiva de la imagen percibida de Acapulco como destino turístico de sol y playa. 
Los resultados permiten ofrecer un grupo de implicaciones empresariales en torno a la promoción y gestión del destino turístico. Dada la creciente competencia entre los destinos de sol y playa, así como las nuevas demandas del turista, han de incorporarse nuevas estrategias competitivas, es decir, atender y cuidar la conservación de su belleza natural y paisajística, destacar las fortalezas referidas en las acciones promocionales y, en su caso, asegurar una mejor gestión medioambiental que resuelva las debilidades detectadas y percibidas por los turistas. Lo aquí encontrado puede ayudar a Acapulco a comprobar su imagen actual y compararla con sus competidores en el mercado nacional, con la posición que pretende proyectar (por parte de la oferta) y con la imagen que realmente perciben los turistas nacionales (por parte de la demanda). Esta comparación debe permitir a los gestores del destino considerar las diferencias entre sus imágenes proyectadas y percibidas por los viajeros o turistas nacionales para planear sus estrategias competitivas, creando y proyectando una imagen basada en la calidad de sus atributos naturales y medioambientales, en concordancia con las tendencias globales en torno a la sustentabilidad de los destinos turísticos.

\section{Fuentes consultadas}

Anderson, J. C. y D. W. Gerbing (1988). "Structural Equation Modeling in Practice: A Review and Recommended Two-Step Approach”. Psychological Bulletin, 103 (3), marzo, 411-423.

Andreu, L., E. Bigné y Ch. Cooper (2000). "Projected and Perceived Image of Spain as a Tourist Destination for British Travellers”. Journal of Travel \& Tourism Marketing, 9 (4), 47-66.

Bagozzi, R. P. y Y. Yi (1988). “On the Evaluation of Structural Equation Models”. Journal of the Academy of Marketing Science, 16 (1), 74-94.

Baloglu, S. (2001). "Image Variations of Turkey by Familiarity Index: Informational and Experiential Dimensions”. Tourism Management, 22 (2), 127133.

y D. Brinberg (1997). “Affective Images of Tourism Destinations”. Journal of Travel Research, 35 (4), 11-15. y K. W. McCleary (1999). "A Model of Destination Image Formation”. Annals of Tourism Research, 26 (4), 868-897. 
y Mehmet Mangaloglu (2001). “Tourism Destination Images of Turkey, Egypt, Greece, and Italy as Perceived by us-Based Tour Operators and Travel Agents”. Tourism Management, 22 (1), 1-9.

Beerli, A. y J. D. Martín (2004a). “Tourists' Characteristics and the Perceived Image of Tourist Destinations: A Quantitative Analysis. A Case Study of Lanzarote, Spain”. Tourism Management, 25 (5), 623-636.

(2004b). "Factors Influencing Destination Image”. Annals of Tourism Research, 31 (3), 657-681.

, J. D. Martín y S. Moreno (2003). “Los agentes que conforman la imagen de los destinos turísticos” [en línea]. I Coloquio Predoctoral Europeo de Turismo y Ocio, ESADE-IMHI (Cornell-essec), 3-4 de abril. Disponible en: http://www.esade.edu/cedit2003/pdfs/morenosergio.pdf

Bigné, J. E. et al. (2008). "Investigación internacional en marketing turístico: análisis de contenido sobre temas y metodologías”. Pasos. Revista de Turismo y Patrimonio Cultural, 6 (3), 391-389.

e I. Sánchez (2001). "Evaluación de la imagen de destinos turísticos: Una aplicación metodológica en la Comunidad Valenciana”. Revista Europea de Dirección y Economía de la Empresa, 10 (3), 189-200.

Bonn, Mark A., S. M. Joseph y Mo Dai (2005). "International versus Domestic Visitors: An Examination of Destination Image Perceptions”. Journal of Travel Research, 43 (3), 294-301.

Camisón, C. y V. Monfort (1998). "Estrategias de reposicionamiento para destinos turísticos maduros: El caso de la Costa Blanca”. Estudios Turísticos, $135,5-28$.

Castaño, J. M., A. Moreno y A. Crego (2006). "Factores psicosociales y formación de imágenes en el turismo urbano: un estudio de caso sobre Madrid”. Pasos. Revista de Turismo y Patrimonio Cultural, 4 (3), 287-299.

Chen, J. S. y C. H. C. Hsu (2000). “Measurement of Korean Tourists' Perceived Images of Overseas Destinations”. Journal of Travel Research, 38 (4), 411-416.

Chon, Kye-Sung (1991). “Tourism Destination Image Modification Process: Marketing Implications”. Tourism Management, 12 (1), 68-72.

Court, B. y R. A. Lupton (1997). "Customer Portfolio Development: Modeling Destination Adopters, Inactives and Rejecters”. Journal of Travel Research, 36 (1), 35-43. 
Del Barrio García, S., T. Luque Martínez y M. Á. Rodríguez Molina (2009). “La modelización de la imagen de ciudad desde la perspectiva de los líderes de opinión externos”. Eure, 35 (106), 9-28.

Dolnicar, S. y B. Grün (2013). “'Translating’ between Survey Answer Formats”. Journal of Business Research, 66 (9), 1298-1306.

Domínguez, P., R. Valdés y M. C. Morfín (2004). “Los medios de comunicación y su impacto en la formación de la imagen de un destino turístico: La opinión del mercado europeo sobre México” [en línea]. XIII Simposio Internacional de Turismo y Ocio ESADE-CEDIT. Disponible en: http:// www.esade.es/cedit2004/pdfs/17_Dominguez.pdf

Echtner, Ch. M. y J. R. Brent Ritchie (2003). "The Meaning and Measurement of Destination Image”. The Journal of Tourism Studies, 14 (1), 37-48.

Ekinci, Yuksel y S. Hosany (2006). "Destination Personality: An Application of Brand Personality to Tourism Destinations”. Journal of Travel Research, 45 (2), 127-139.

Fornell, C. y D. F. Larcker (1981). "Evaluating Structural Equation Models with Unobservable Variables and Measurement Error”. Journal of Marketing Research, 18 (1), 39-50.

Gartner, William C. (1993). “Image Formation Process”, en M. Uysal y D. Fesenmaier (eds.). Communication and Channel Systems in Tourism Marketing. Nueva York: The Haworth Press, 191-216.

Goodrich, Jonathan N. (1977). "Benefit Bundle Analysis: An Empirical Study of International Travelers". Journal of Travel Research, 16 (2), 6-9.

- (1978). "A New Approach to Image Analysis through Multidimensional Scaling”. Journal of Travel Research, 16 (3), 3-7.

Govers, R. (2005). Virtual Tourism Destination Image: Glocal Identities Constructed, Perceived and Experienced. Róterdam: Erasmus Research Institute of Management.

Grosspietsch, M. (2006). Perceived and projected images of Rwanda: visitor and international tour operator perspectives. Tourism Management, $27,225-234$.

Hair, J. H. et al. (2006). Multivariate Data Analysis. 6a ed. Nueva Jersey: Prentice Hall.

Hong, Sung-Kwon et al. (2006). “The Roles of Categorization, Affective Image 
and Constraints on Destination Choice: An Application of the NMNL Model”. Tourism Management, 27 (5), 750-761.

Hosany, S., Ekinci, Y. y Uysal, M. (2007). Destination Image and Destination Personality. International Journal of Culture, Tourism and Hospitality Research, 1(1), 62-81.

Y. Ekinci y M. Uysal (2006). "Destination Image and Destination Personality: An Application of Branding Theories to Tourism Places”. Journal of Business Research, 59 (5), 638-642.

Hsu, C. H. C., K. Wolfe y Soo K. Kang (2004). "Image Assessment for a Destination with Limited Comparative Advantages”. Tourism Management, 25 (1), 121-126.

Hu, Yangzhou y J. R. Brent Ritchie (1993). "Measuring Destination Attractiveness: A Contextual Approach”. Journal of Travel Research, 32 (2), 25-34.

Hui, Tak Kee y Tai Wai David Wan (2003). “Singapore's Image as a Tourist Destination”. International Journal of Tourism Research, 5 (4), 305-313. Joppe, M., D. W. Martin y J. Waalen (2001). “Toronto’s Image as a Destination: A Comparative Importance-Satisfaction Analysis by Origin of Visitor”. Journal of Travel Research, 39 (1), 252-260.

Kale, Sudhir H. y Katherine M. Weir (1986). "Marketing Third World Countries to the Western Traveler: The Case of India”. Journal of Travel Research, 25 (2), 2-7.

Kim, Hong-Bumm (1998). "Perceived Attractiveness of Korean Destinations". Annals of Tourism Research, 25 (2), 340-361.

Kim, Hyounggon y S. L. Richardson (2003). "Motion Picture Impacts on Destination Images”. Annals of Tourism Research, 30 (1), 216-237.

Konecnik, M. (2004). "Evaluating Slovenia's Image as a Tourism Destination: A Self-Analysis Process Towards Building a Destination Brand”. Journal of Brand Management, 11 (4), 307-316.

(2005). "Slovenia as a Tourism Destination: Differences in Image Evaluations Perceived by Tourism Representatives from Closer and More Distant Markets”. Economic and Business Review, 7 (3), 261-282.

Konecnik, M. y W. C. Gartner (2007). "Customer-Based Brand Equity for a Destination”. Annals of Tourism Research, 34 (2), 400-421. 
Kozak, M. (2001). "Comparative Assessment of Tourist Satisfaction with Destinations Across Two Nationalities”. Tourism Management, 22 (4), 391401.

Lee, Jin Soo et al. (2010). "Understanding How Consumers View Green Hotels: How a Hotel's Green Image Can Influence Behavioural Intentions”. Journal of Sustainable Tourism, 18 (7), 901-914.

López, D. (2014). Los recursos turísticos. Evaluación, ordenación y planificación turística. Estudio de casos. Valencia: Tirant lo Blanch.

Luque, T. et al. (2004). "La modelización de la imagen de Granada desde la perspectiva de los líderes de opinión externos” [en línea]. XVI Encuentro de Profesores Universitarios de Marketing, Alicante, 22, 23 y 24 de septiembre. Disponible en: http://www.epum2004.ua.es/aceptados/257.pdf

Medina, C., M. Rey y R. Rufín (2010). “Imagen de los destinos turísticos urbanos y lealtad del turista... iActitud o comportamiento?” Estudios y Perspectivas en Turismo, 19 (2), 279-298.

Murphy, L. (1999). “Australia’s Image as a Holiday Destination-Perceptions of Backpacker Visitors". Journal of Travel and Tourism Marketing, 8 (3), 21-45.

Nadeau, J. et al. (2008). "Destination in a Country Image Context". Annals of Tourism Research, 35 (1), 84-106.

O’Leary, Sinéad y J. Deegan (2003). "People, Pace, Place: Qualitative and Quantitative Images of Ireland as a Tourism Destination in France”. Journal of Vacation Marketing, 9 (3), 213-226.

Phelps, A. (1986). "Holiday Destination Image-The Problem of Assessment: An Example developed in Menorca”. Tourism Management, 7 (3), 168-180.

Pike, S. D. (2003). "The Use of Repertory Grid Analysis to Elicit Salient Short Break Holiday Attributes in New Zealand”. Journal of Travel Research, 41 (3), 315-319.

(2007). "Repertory Grid Analysis in Group Settings to Elicit Salient Destination Image Attributes”. Current Issues in Tourism, 10 (4), 378-392. y C. Ryan (2004). "Destination Positioning Analysis through a Comparison of Cognitive, Affective, and Conative Perceptions”. Journal of Travel Research, 42 (4), 333-342. 
Prados, E. (2002). "Medio ambiente y turismo, dos realidades sinérgicas en el nuevo milenio". Derecho y Medio Ambiente. Revista Jurídica para el Desarrollo Sostenible, 3 (9), 53-95.

Rittichainuwat, Bongkosh Ngamsom, Hailin Qu y Tom J. Brown (2001). "Thailand's International Travel Image: Mostly Favorable". The Cornell Hotel and Restaurant Administration Quarterly, 42 (2), 82-95.

San Martín, H. e I. Rodríguez del Bosque (2008). "Exploring the CognitiveAffective Nature of Destination Image and the Role of Psychological Factors in its Formation”. Tourism Management, 29 (2), 263-277.

---------, I. Rodríguez del Bosque y R. Vázquez (2006). “Análisis de la imagen en turismo mediante técnicas estructuradas y no estructuradas: implicaciones competitivas para los destinos turísticos”. Revista Asturiana de Economía, 35, 69-91.

San Martín Rebolloso, F. y M. P. Salcedo (2007). “Turismo, sustentabilidad y certificación: Un reto global”. Revista del Centro de Investigación. Universidad La Salle, 7 (27), 77-91.

Sánchez-García, I. et al. (2007). "The Image of a Destination as a Key Variable in the Segmentation of Tourists: An Analysis of Its Influence on Behavior” [en línea]. Disponible en: http://www.escp-eap.eu/conferences/marketing/2007_cp/Materiali/Paper/Fr/SanchezGarcia_SanzBlas_GonzalezM_MiquelR.pdf

Sancho, F. y J. Álvarez (2010). "Tourism Destination Image and Motivations: The Spanish Perspective of Mexico". Journal of Travel and Tourism Marketing, 27 (4), 349-360.

Solís, M. M. et al. (2014). "Análisis exploratorio de la fidelidad y los principales conceptos relacionados en turismo: el caso de Ixtapa/ZihuatanejoMéxico". Revista de Estudios Empresariales, 1, 90-112.

Tasci, Asli D. A., Gartner, W. C. y Cavusgil, S. Tamer (2007). Measurement of destination brand bias using a quasi-experimental design. Tourism Management, 28, 1529-1540.

Tapachai, N. y R. Waryszak (2000). "An Examination of the Role of Beneficial Image in Tourist Destination Selection". Journal of Travel Research, 39 (1), 37-44. 
Vera, J. F. y C. J. Baños (2010). "Renovación y reestructuración de los destinos turísticos consolidados del litoral: las prácticas recreativas en la evolución del espacio turístico”. Boletín de la Asociación de Geógrafos Españoles, 53 (2), 329-353.

Vogt, Ch. A. y K. L. Andereck (2003). "Destination Perceptions Across a Vacation”. Journal of Travel Research, 41 (4), 348-354.

White, Ch. J. (2002). "Emotions, Gender and Destination Visitation Intentions" [en línea]. I Coloquio Predoctoral Europeo de Turismo y Ocio, ESADEIMHI (Cornell-essec), 3-4 de abril. Disponible en: http://www.esade.es/ cedit2003/pdfs/whitechristopher.pdf

Wong, K. W. (2004). Perceived tourism destination image of western China: Evidence from Hong Kong residents (Doctoral dissertation, The Hong Kong Polytechnic University). 